\title{
Technique de repérage échographique du premier anneau trachéal
}

\section{Ultrasound technique to locate the first tracheal ring}

\author{
Farid Arezki, MD • Galina Netcheva, MD • \\ Ulrich Johann, MD · Andréas Eichler, MD
}

Received: 6 July 2009/ Accepted: 4 September 2009/Published online: 6 October 2009

(C) Canadian Anesthesiologists' Society 2009

\section{Au rédacteur en chef,}

La ponction trachéale lors de la réalisation d'une trachéotomie percutanée est parfois difficile lorsque la palpation ne permet pas le repérage des différents éléments anatomiques. ${ }^{1} \mathrm{C}$ 'est un problème qui se pose également lors d'une intubation rétrograde ou une ventilation transtrachéale (jet ventilation) où le succès rapide de la ponction trachéale peut être vital chez le patient qu'on n'arrive ni à intuber et ni à ventiler. ${ }^{2}$

Chez douze patients devant bénéficier d'une trachéotomie percutanée, un repérage échographique en mode B est réalisé avec pour objectif une ponction trachéale entre le premier et le deuxième anneau trachéal. Le matériel utilisé est la machine MicroMaxx de SonoSite (Villebon sur Yvette, France) et une sonde linéaire de 13-6 MHz (sonde utilisée pour le repérage vasculaire et nerveux). La sonde est d'abord placée au contact du cartilage thyroïde, orientée parallèlement à l'axe des deux épaules de façon à avoir une image en coupe transversale. La sonde est ensuite déplacée dans le même plan de haut en bas du cartilage thyroïde du larynx jusqu'en sus-sternal. Une analyse des différentes catégories d'images échographiques obtenues est réalisée tout en s'aidant de la palpation quand celle-ci semble fiable.

Dans tous les cas, trois types d'images ont été successivement identifiés et correspondent aux niveaux suivants :

1- Le cartilage thyroïde : aspect en $\mathrm{V}$ inversé avec un sommet émoussé suivi d'une ombre acoustique (noir) (Fig. 1).

F. Arezki, MD ( $₫) \cdot$ G. Netcheva, MD · U. Johann, MD .

A. Eichler, MD

C.H. Robert-Pax, Sarreguemines, France

e-mail: farid.arezki@ch-sarreguemines.fr

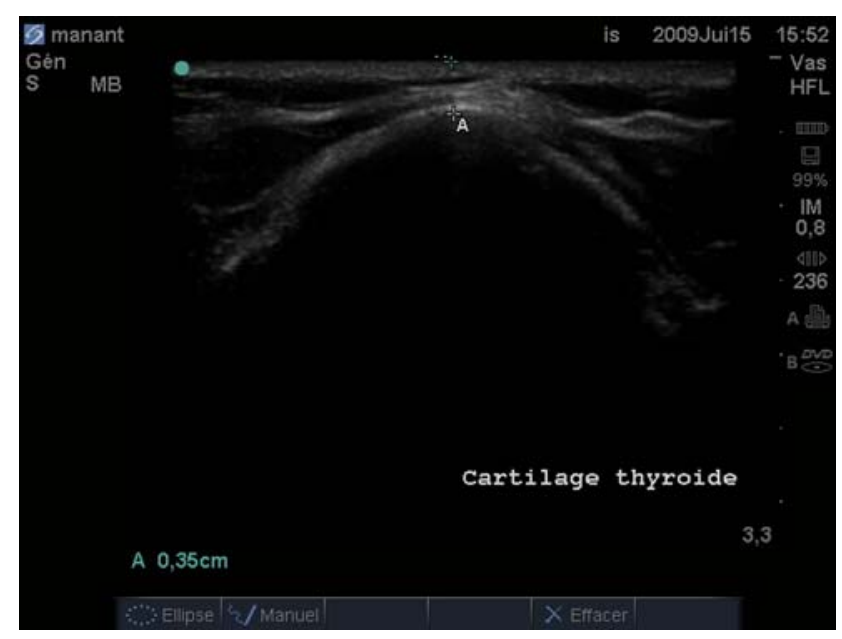

Fig. 1 Cartilage thyroïde

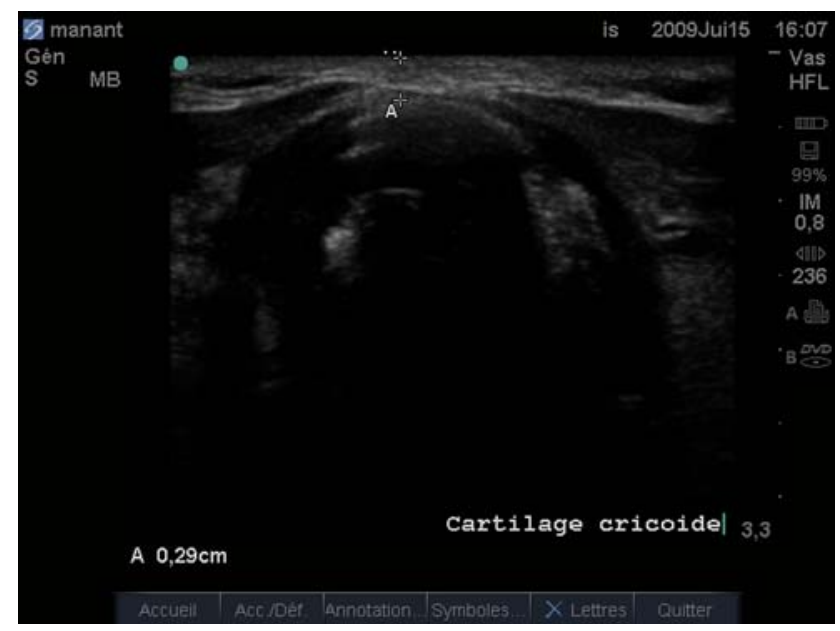

Fig. 2 Cartilage cricoïde 


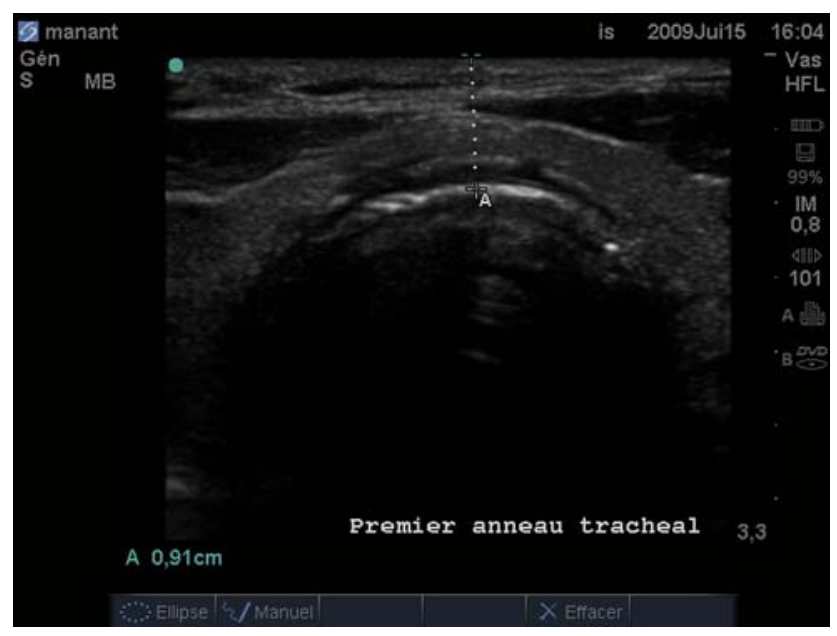

Fig. 3 Premier anneau trachéal

2- Le cartilage cricoïde : aspect d'arc hyperéchogène (blanc) situé à la même profondeur que le cartilage thyroïde (Fig. 2).

3- Le premier anneau trachéal : aspect hypoéchogène suivi d'une ligne hyperéchogène dont la localisation est plus profonde que l'arc hyperéchogène du cartilage thyroïde. Cette image est parfois répétée (échos retardataires ou de réverbération: échos parasites retardés espacés régulièrement) (Fig. 3).

Cette technique de repérage permet une ponction rapide au niveau choisi tout en restant bien médian améliorant ainsi la sécurité du geste. En dehors de la trachéotomie percutanée, elle pourrait avoir sa place dans l'intubation difficile prévue et imprévue. ${ }^{3}$

\section{Conflit d'intérêt Aucun.}

\section{Références}

1. Muhammad JK, Patton DW, Evans RM, Major E. Percutaneous dilatational tracheostomy under ultrasound guidance. Br J Oral Maxillofac Surg 1999; 37: 309-11.

2. Orr JA, Stephens RS, Mitchell VM. Ultrasound-guided localisation of the trachea. Anaesthesia 2007; 62: 972-3.

3. Gerig HG, Schnider T, Heidegger T. Prophylactic percutaneous transtracheal catheterisation in the management of patients with anticipated difficult airways: a case series. Anaesthesia 2005; 60: $801-5$. 